\title{
The Lomax-Exponential Distribution, Some Properties and Applications
}

\author{
Nasrin Hami Golzar ${ }^{\dagger}$, Masoud Ganji ${ }^{\dagger, *}$ and Hossein Bevrani ${ }^{\ddagger}$ \\ † University of Mohaghegh Ardabili \\ $¥$ University of Tabriz
}

Received: 1/21/2016 Approved: 1/28/2017

\begin{abstract}
The exponential distribution is a popular model in applications to real data. We propose a new extension of this distribution, called the Lomax-exponential distribution, which presents greater flexibility to the model. Also there is a simple relation between the Lomax-exponential distribution and the Lomax distribution. Results for moment, limit behavior, hazard function, Shannon entropy and order statistic are provided. To estimate the model parameters, the method of maximum likelihood and Bayse estimations are proposed. Two data sets are used to illustrate the applicability of the Lomax-exponential distribution.
\end{abstract}

Keywords. T-X family; Lomax distribution; Shannon entropy; simulation study.

MSC 2010: 62E15; 60E05.

\section{Introduction}

The exponential and Lomax distributions are two important distributions in statistical practice. They possess several important statistical properties

Corresponding author 
and exhibit great mathematical tractability. History of exponential density function has been obtained in Balakrishnan and Basu (1995). Gupta and Kundu (1999a) introduced generalized exponential density function. Then Gupta and Kundu (1999b) derived some properties of generalized exponential density function. The Lomax distribution, conditionally also called the Pareto Type II distribution, is used in business, economics, actuarial science and etc. It is essentially a Pareto distribution that has been shifted so that its support begins at zero. An extended Lomax distribution was introduced by Lemonte and Cordeiro (2011).

Alzaatreh et al. (2013a) proposed the T-X families of distributions. These families of distributions were used to generate a new class of distributions which offer more flexibility in modeling a variety of data sets.

Various distributions can be driven by using this method. The gammahalf normal and the gamma-normal distributions were introduced by Alzaatreh and Knight (2013) and Alzaatreh et al. (2014) respectively. Also the gamma-Pareto and Weibull-Pareto distributions were studied by Alzaatreh et al. (2012) and Alzaatreh et al. (2013b) respectively. The gamma-uniform and logistic-uniform distributions were introduced by Torabi and Montazeri $(2012,2014)$. In this paper new distribution, Lomax-exponential will be introduced by using of the method of T-X family.

Let $F(x)$ be the cumulative distribution function (c.d.f.) of any random variable $X$. Also let $r(t)$ and $R(t)$ be the probability density function (p.d.f.) and c.d.f. of a random variable $T$ defined on $(0, \infty)$ respectively. The c.d.f. of the T-X family of distributions defined by Alzaatreh et al. (2013a) is given by

$$
G(x)=\int_{0}^{\frac{F(x)}{1-F(x)}} r(t) d t=R\left(\frac{F(x)}{1-F(x)}\right) .
$$

When $X$ is a continuous random variable, the probability density function of the T-X family is

$$
g(x)=\frac{f(x)}{(1-F(x))^{2}} r\left(\frac{F(x)}{1-F(x)}\right) .
$$

Let a random variable $T$ follows the Lomax distribution with p.d.f. and c.d.f. respectively for $t>0, k, a>0$ as 


$$
r(t)=\frac{k a^{k}}{(t+a)^{k+1}}
$$

and

$$
R(t)=1-\left(\frac{a}{t+a}\right)^{k} .
$$

Then $g(x)$ in (2) leads to the Lomax-X family with p.d.f.

$$
g(x)=\frac{k a^{k} f(x)(1-F(x))^{k-1}}{[F(x)+a\{1-F(x)\}]^{k+1}} .
$$

The Lomax-exponential distribution (LED for short) is obtained if $X$ follows the exponential distribution with parameter $s$.

The corresponding c.d.f. and p.d.f. of $X$ are respectively defined for $k, a, s>0$ and $x>0$ as

$$
G(x)=1-\left(\frac{a}{e^{s x}+a-1}\right)^{k}
$$

and

$$
g(x)=\frac{s k a^{k} e^{s x}}{\left(e^{s x}+a-1\right)^{k+1}} .
$$

When $k=a=1$, the LED reduces to the exponential distribution. Then the LED is a generalized of the exponential disreibution.

\section{Properties of the Lomax-Exponential Distribu- tion}

In this section some general properties of the LED will be addressed including the limiting behavior, Shannon entropy, quantile function and etc. Lemmas 1 and 2 give the relations between the LED, Lomax and Pareto type 1 distributions.

Lemma 1. If a random variable $X$ follows the LED with parameters $(k, a, s)$, then the random variable $Y=e^{s X}-1$ follows the Lomax distribution with 
parameters $(k, a)$. Also $Y=e^{s X}+a-1$ follows the Pareto type 1 distribution with parameters $(k, a)$.

Proof. The result follows by using the transformation technique.

Lemma 2. If a random variable $X$ follows the Lomax distribution with parameters $(k, a)$, then the random variable $V=\frac{1}{s} \log (X+1)$ follows the LED with parameters $(k, a, s)$.

Proof. The result follows by using the transformation technique.

In Figure 1, various graphs of $g(x)$ are provided. The p.d.f. of LED may take on a variety of shapes for different values of $k, a$ and $s$. It can be right-skewed, approximately symmetric or reversed-J shape. The limit of the p.d.f. of LED as $x \rightarrow 0$ and $x \rightarrow \infty$ are $\frac{s k a^{k}}{a^{k+1}}$ and 0 , respectively. The Figure 1 confirms these limits.

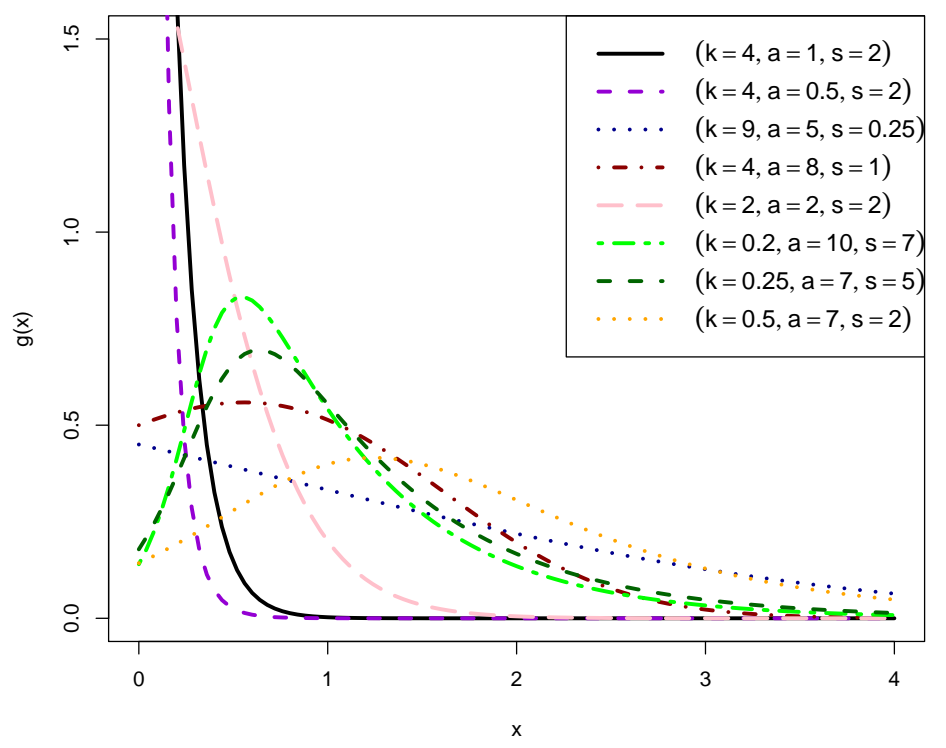

Figure 1. The p.d.f. of LED for various value of $k, a$ and $s$

The hazard function associated with the LED is

$$
h(x)=\frac{g(x)}{1-G(x)}=\frac{s k e^{s x}}{e^{s x}+a-1} .
$$


In Figure 2, various graphs of $h(x)$ are provided for various values of $k, a$ and $s$. It is clear that when $a>1$, the hazard function is increasing, when $a<1$, the hazard function is decreasing and when $a=1$, the hazard function is fixed $(=s k)$. The limits of the hazard function of the LED as $x \rightarrow 0$ and $x \rightarrow+\infty$ are $\frac{s k}{a}$ and $s k$, respectively.

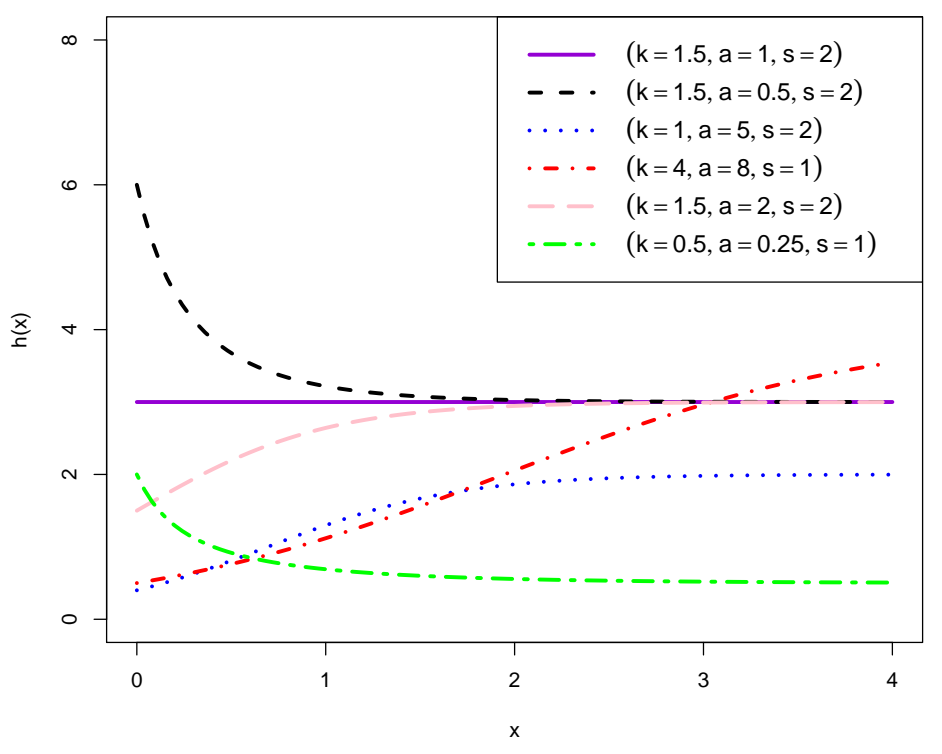

Figure 2. The hazard function of LED for various value of $k, a$ and $s$

Theorem 1. The LED has a unique mode at $x=x_{0}$. When $a \geqslant k+1$, the mode is $x_{0}=\frac{1}{s} \log \frac{a-1}{k}$ and when $a<k+1$ the mode is $x_{0}=0$.

Proof. The mode of the LED can be obtained by taking the derivative of $g(x)$ with respect to $x,\left(g^{\prime}\right)$ and by setting it to zero. It is easy to see $g^{\prime}>0$ for $0<x<\frac{1}{s} \log \frac{a-1}{k}$ and $g^{\prime}<0$ for $x>\frac{1}{s} \log \frac{a-1}{k}$.

\subsection{Quantile Function}

The quantile function of the LED, $Q(\lambda), 0<\lambda<1$ is obtained by solving $G(Q(\lambda))=\lambda$. It implies that 


$$
Q(\lambda)=\frac{1}{s} \log \left(\frac{a}{\sqrt[k]{1-\lambda}}-a+1\right) .
$$

The median of the LED is obtained as

$$
\text { Median }=Q\left(\frac{1}{2}\right)=\frac{1}{s} \log \left(\frac{a}{\sqrt[k]{\frac{1}{2}}}-a+1\right)
$$

\subsection{Moment}

The moment generating function for the LED is given by

$$
M_{X}(t)=E\left(e^{t X}\right)=\int_{0}^{\infty} e^{t x} g(x) d x=s k a^{k} \int_{0}^{\infty} \frac{e^{(t+s) x}}{\left(e^{s x}+a-1\right)^{k+1}} d x
$$

By defining $y=e^{s x}+a-1$

$$
M_{X}(t)=k a^{k} \int_{a}^{\infty} \frac{(y-a+1)^{\frac{t}{s}}}{y^{k+1}} d y .
$$

Using Taylor expansion

$$
\begin{aligned}
(y-a+1)^{\frac{t}{s}} & =y^{\frac{t}{s}}+\frac{t}{s} y^{\frac{t}{s}-1}(1-a)+\frac{\frac{t}{s}\left(\frac{t}{s}-1\right) y^{\frac{t}{s}-2}(1-a)^{2}}{2}+\cdots \\
& \simeq y^{\frac{t}{s}}+\frac{t}{s} y^{\frac{t}{s}-1}(1-a) .
\end{aligned}
$$

Then

$$
\begin{aligned}
M_{X}(t) & =k a^{k} \int_{a}^{\infty} \frac{(y-a+1)^{\frac{t}{s}}}{y^{k+1}} d y \\
& \simeq k a^{k} \int_{a}^{\infty} \frac{y^{\frac{t}{s}}+\frac{t}{s} y^{\frac{t}{s}-1}(1-a)}{y^{k+1}} d y \\
& =k\left\{\frac{a^{\frac{t}{s}}}{k-\frac{t}{s}}+\frac{(1-a) t a^{\frac{t}{s}}-1}{s\left(k+1-\frac{t}{s}\right)}\right\},
\end{aligned}
$$


provided $t<k s$. The derivative of (10) with respect to $t$ is given by

$$
\begin{aligned}
\frac{\partial}{\partial t} M_{X}(t)= & \frac{k}{s}\left(\frac{a^{\frac{t}{s}}\left(k-\frac{t}{s}\right) \log a+a^{\frac{t}{s}}}{\left(k-\frac{t}{s}\right)^{2}}\right) \\
& +\frac{k}{s}\left[\frac{(1-a)\left\{\left(s a^{\frac{t}{s}-1}+t a^{\frac{t}{s}-1} \log a\right)\left(k+1-\frac{t}{s}\right)+t a^{\frac{t}{s}-1}\right\}}{\left(k+1-\frac{t}{s}\right)^{2}}\right] .
\end{aligned}
$$

The first non-central moment follows by setting $\mathrm{t}=0$,

$$
E_{L E D}(X)=\frac{k \log a+1}{s k}+\frac{k(1-a)}{a s(k+1)} .
$$

By defining $k=a=1$ in (11), the first non-central moment of the exponential distribution is obtained as

$$
E_{L E D}(X)=\frac{1}{s} .
$$

Simultaneously, the equation (11) works well in Subsection 2.7.

\subsection{Shannon Entropy}

The entropy of a random variable $X$ is a measure of variation of uncertainty. Shannon entropy for a random variable $X$ with p.d.f. $g(x)$ is defined as $E(-\log g(X))$ (Shannon 1948). The following theorem gives the Shannon entropy of the LED.

Theorem 2. Let $X$ be a random variable that follows the LED given in (7). Then the Shannon entropy of $X$ is

$$
\eta_{x}=\log \frac{1}{s k}-\frac{k(1-a)}{a(k+1)}+1 .
$$

Proof. For LED, the Shannon entropy is given by

$$
\begin{aligned}
\eta_{x} & =E_{L E D}\{-\log g(X)\} \\
& =-E_{L E D}\left\{\log s+\log k+k \log a+s X-(k+1) \log \left(e^{s X}+a-1\right)\right\} \\
& =-\log s-\log k-k \log a-s E_{L E D}(X)+(k+1) E_{L E D}\left(\log \left\{e^{s X}+a-1\right)\right\} .
\end{aligned}
$$


Using Lemma 1, one can get

$$
E_{L E D}\left(\log \left(e^{s X}+a-1\right)\right)=\log a+\frac{1}{k} .
$$

The result can be obtained by (11).

\subsection{Order Statistics}

The density function of the $i$ th order statistic, $X_{i: n}$ for a random sample of size $n$ drawn from (7) from Arnold et al. (2008) is

$$
\begin{aligned}
f_{X_{i: n}}(x)= & \frac{n !}{(n-i) !(i-1) !} g(x) G(x)^{i-1}(1-G(x))^{n-i} \\
= & \frac{\Gamma(n+1)}{\Gamma(i) \Gamma(n+1-i)} \cdot \frac{s k a^{k} e^{s x}}{\left(e^{s x}+a-1\right)^{k+1}}\left(1-\frac{a^{k}}{\left(e^{s x}+a-1\right)^{k}}\right)^{i-1} \\
& \times\left(\frac{a^{k}}{\left(e^{s x}+a-1\right)^{k}}\right)^{n-i} \\
= & \frac{\Gamma(n+1)}{\Gamma(i) \Gamma(n+1-i)} \cdot \frac{s k a^{k} e^{s x}}{\left(e^{s x}+a-1\right)^{k+1}}\left(\frac{a^{k}}{\left(e^{s x}+a-1\right)^{k}}\right)^{n-i} \\
& \times \frac{\left\{\left(e^{s x}+a-1\right)^{k}-a^{k}\right\}^{i-1}}{\left(e^{s x}+a-1\right)^{k i-k}} .
\end{aligned}
$$

Using of the binomial expansion and some calculations, we obtain

$$
\begin{aligned}
f_{X_{i: n}}(x) & =\frac{\Gamma(n+1)}{\Gamma(i) \Gamma(n+1-i)} \cdot \frac{s k a^{k} e^{s x}}{\left(e^{s x}+a-1\right)^{k+1}} \sum_{j=0}^{i-1} \frac{(-1)^{i-j-1}\left(\begin{array}{c}
i-1 \\
j
\end{array}\right) a^{(n-j-1) k}}{\left(e^{s x}+a-1\right)^{k(n-j-1)}} \\
& =\sum_{j=0}^{i-1} \frac{\Gamma(n+1)\left(\begin{array}{c}
i-1 \\
j
\end{array}\right)(-1)^{i-j-1}}{\Gamma(i) \Gamma(n+1-i)(n-j)} \cdot \frac{s k(n-j) a^{(n-j) k} e^{s x}}{\left(e^{s x}+a-1\right)^{(n-j) k+1}} \\
& =\sum_{j=0}^{i-1} \eta_{n, j, i} g_{(n-j) k, a, s}(x),
\end{aligned}
$$

where

$$
\eta_{n, j, i}=\frac{\Gamma(n+1)\left(\begin{array}{c}
i-1 \\
j
\end{array}\right)(-1)^{i-j-1}}{\Gamma(i) \Gamma(n+1-i)(n-j)}
$$


and $g_{(n-j) k, a, s}(x)$ is the p.d.f. of LED with parameters $(n-j) k, a$ and s. The first non-central moment of $X_{i: n}$ is obtained by (11) as

$$
E_{X_{i: n}}(X)=\sum_{j=0}^{i-1} \eta_{n, j, i}\left\{\frac{(n-j) k \log a+1}{(n-j) s k}+\frac{k(n-j)(1-a)}{a s((n-j) k+1)}\right\} .
$$

The density of the first order statistic from (12) is

$$
f_{X_{1: n}}(x)=\frac{s n k a^{n k} e^{s x}}{\left(e^{s x}+a-1\right)^{n k+1}} .
$$

Then the first non-central moment of $X_{1: n}$ is

$$
E_{X_{1: n}}(X)=\frac{n k \log a+1}{n s k}+\frac{n k(1-a)}{a s(n k+1)} .
$$

The result in (13) indicates that the first order statistic follows the LED with the parameters $n k, a$ and $s$, then the properties of the first order statistic can be found easily.

\subsection{Maximum Likelihood Estimation}

Let $X_{1}, \ldots, X_{n}$ be a random sample of size $n$ taken from the LED. The log-likelihood function for the LED by using (7) is given by

$$
\begin{aligned}
\log L(k, a, s)= & n \log s+n \log k+n k \log a+s \sum_{i=1}^{n} x_{i} \\
& -\sum_{i=1}^{n} \log \left(e^{s x_{i}}+a-1\right)^{k+1} .
\end{aligned}
$$

The derivatives of (14) with respect to $k, a$ and $s$, respectively, are given by

$$
\frac{\partial \log L}{\partial k}=\frac{n}{k}+n \log a-\sum_{i=1}^{n} \log \left(e^{s x_{i}}+a-1\right),
$$




$$
\frac{\partial \log L}{\partial a}=\frac{n k}{a}-\sum_{i=1}^{n} \frac{k+1}{e^{s x_{i}}+a-1}
$$

and

$$
\frac{\partial \log L}{\partial s}=\frac{n}{s}+\sum_{i=1}^{n} x_{i}-\sum_{i=1}^{n} \frac{(k+1) x_{i} e^{s x_{i}}}{e^{s x_{i}}+a-1} .
$$

Now by setting (15), (16) and (17) to zero and solving them simultaneously, one can get the Maximum Likelihood Estimate (MLE) of $k, a$ and $s$ which are denoted by $\hat{k}, \hat{a}$ and $\hat{s}$.

For interval estimation of the model parameters, we require the $3 \times 3$ observed information matrix $J(\Theta)=J_{r s}$ (for $\left.r, s=k, a, s\right)$ (A matrix of second partial

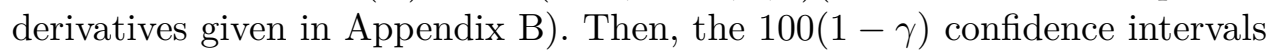
for $k, a$ and $s$ are given by $\hat{k} \pm z_{\frac{\gamma}{2}} \sqrt{\operatorname{var}(\hat{k})}, \hat{a} \pm z_{\frac{\gamma}{2}} \sqrt{\operatorname{var}(\hat{a})}$ and $\hat{s} \pm z_{\frac{\gamma}{2}} \sqrt{\operatorname{var}(\hat{s})}$, respectively, where the $\operatorname{var}(\cdot)^{\prime}$ 's denote the diagonal elements of $J(\Theta)^{-1}$ corresponding to the model parameters, and $z_{\frac{\gamma}{2}}$ is the quantile $\left(1-\frac{\gamma}{2}\right)$ of the standard normal distribution.

We evaluated the performance of the maximum likelihood method for estimating the LED parameters using simulation for a total of thirteen parameter combinations and the process is repeated 300 times. We used "nlminb"function in $R$ software for optimization. The MLEs, $0.95 \%$ confidence interval estimation and root of mean squared error (RMSE) of the parameter estimates were listed in Table 1 . We note that the MLE method performs acceptability for estimating the model parameters.

\subsection{Bayes Estimation}

In this subsection, we obtain Bayes estimates of the parameters of the LED using quadratic loss function. Tierney and Kadane (1986) gave an approximate form for the evaluation of the Bayes estimates of $\phi(\lambda)$ by writing the two expressions

$$
l(\lambda)=\frac{1}{n} \ln q(\lambda \mid x)
$$

and 
Table 1. MLEs, Confidence Intervals and the root of MSE (RMSE) for various parameter values

\begin{tabular}{|c|c|c|c|c|c|c|c|c|}
\hline \multicolumn{3}{|c|}{ Actual values } & \multicolumn{2}{|c|}{ Estimates of $k$} & \multicolumn{2}{|c|}{ Estimates of $a$} & \multicolumn{2}{|c|}{ Estimates of $s$} \\
\hline$k$ & $a$ & $s$ & $\begin{array}{c}\text { Mean } \\
\text { (Conf. Int.) }\end{array}$ & RMSE & $\begin{array}{c}\text { Mean } \\
\text { (Conf. Int.) }\end{array}$ & RMSE & $\begin{array}{c}\text { Mean } \\
\text { (Conf. Int.) }\end{array}$ & RMSE \\
\hline 0.25 & 0.25 & 0.25 & $\begin{array}{c}0.2668 \\
(0.11,0.41)\end{array}$ & 0.0192 & $\begin{array}{c}0.2499 \\
(0.15,0.34)\end{array}$ & 0.0032 & $\begin{array}{c}0.2556 \\
(0.06,0.44)\end{array}$ & 0.0243 \\
\hline 0.25 & 0.25 & 0.5 & $\begin{array}{c}0.2687 \\
(0.19,0.34)\end{array}$ & 0.0213 & $\begin{array}{c}0.2529 \\
(0.15,0.34)\end{array}$ & 0.0044 & $\begin{array}{c}0.5390 \\
(0.36,0.71)\end{array}$ & 0.0670 \\
\hline 0.25 & 0.5 & 0.25 & $\begin{array}{c}0.2933 \\
(0.23,0.35)\end{array}$ & 0.0516 & $\begin{array}{c}0.4842 \\
(0.23,0.72)\end{array}$ & 0.0200 & $\begin{array}{c}0.3036 \\
(0.22,0.37)\end{array}$ & 0.0643 \\
\hline 0.5 & 0.25 & 0.25 & $\begin{array}{c}0.5251 \\
(0.32,0.72)\end{array}$ & 0.0387 & $\begin{array}{c}0.2553 \\
(0.15,0.35)\end{array}$ & 0.0030 & $\begin{array}{c}0.2684 \\
(0.13,0.40)\end{array}$ & 0.0227 \\
\hline 1 & 0.5 & 0.25 & $\begin{array}{c}1.0530 \\
(0.80,1.30)\end{array}$ & 0.0648 & $\begin{array}{c}0.4820 \\
(0.32,0.64)\end{array}$ & 0.0195 & $\begin{array}{c}0.4673 \\
(0.35,0.45)\end{array}$ & 0.2130 \\
\hline 0.75 & 1 & 0.25 & $\begin{array}{c}0.7755 \\
(0.21,1.33)\end{array}$ & 0.0328 & $\begin{array}{c}1.1301 \\
(0.76,1.49)\end{array}$ & 0.0810 & $\begin{array}{c}0.8201 \\
(0.61,1.02)\end{array}$ & 0.6538 \\
\hline 0.25 & 0.75 & 1 & $\begin{array}{c}0.6024 \\
(0.49,0.70)\end{array}$ & 0.4075 & $\begin{array}{c}0.7297 \\
(0.41,1.02)\end{array}$ & 0.0202 & $\begin{array}{c}1.1600 \\
(0.70,1.61)\end{array}$ & 0.1600 \\
\hline 1.5 & 0.25 & 0.25 & $\begin{array}{c}1.4699 \\
(0.94,1.97)\end{array}$ & 0.0300 & $\begin{array}{c}0.2635 \\
(0.03,0.48)\end{array}$ & 0.0135 & $\begin{array}{c}0.3923 \\
(0.19,0.58)\end{array}$ & 0.1423 \\
\hline 0.25 & 1.5 & 0.5 & $\begin{array}{c}0.4650 \\
(0.34,0.58)\end{array}$ & 0.2150 & $\begin{array}{c}1.7133 \\
(1.09,2.33)\end{array}$ & 0.2133 & $\begin{array}{c}0.5666 \\
(0.34,0.78)\end{array}$ & 0.0966 \\
\hline 0.5 & 0.25 & 1.25 & $\begin{array}{c}0.5191 \\
(0.33,0.70)\end{array}$ & 0.0191 & $\begin{array}{c}0.2467 \\
(0.14,0.34)\end{array}$ & 0.0032 & $\begin{array}{c}1.3674 \\
(0.71,2.01)\end{array}$ & 0.1174 \\
\hline 0.5 & 0.25 & 2.25 & $\begin{array}{c}0.5222 \\
(0.31,0.73)\end{array}$ & 0.0222 & $\begin{array}{c}0.2453 \\
(0.15,0.33)\end{array}$ & 0.0046 & $\begin{array}{c}2.4959 \\
(1.11,3.95)\end{array}$ & 0.2459 \\
\hline 1 & 0.25 & 2 & $\begin{array}{c}1.0428 \\
(0.59,1.48)\end{array}$ & 0.0428 & $\begin{array}{c}0.2447 \\
(0.10,0.38)\end{array}$ & 0.0052 & $\begin{array}{c}2.5338 \\
(0.97,4.09)\end{array}$ & 0.5338 \\
\hline 0.5 & 2 & 0.25 & $\begin{array}{c}0.6672 \\
(0.54,0.78)\end{array}$ & 0.1672 & $\begin{array}{c}2.2825 \\
(1.69,2.87)\end{array}$ & 0.2825 & $\begin{array}{c}0.3191 \\
(0.26,0.36)\end{array}$ & 0.0691 \\
\hline
\end{tabular}




$$
l^{*}(\lambda)=l(\lambda)+\frac{1}{n} \ln \phi(\lambda)
$$

So the Bayse estimations of $\phi(\lambda)$ are defined as

$$
\hat{\phi_{B}}(\lambda)=\left(\frac{\operatorname{det} \Omega^{*}}{\operatorname{det} \Omega}\right)^{\frac{1}{2}} \phi\left(\hat{\lambda^{*}}\right) \frac{q\left(\hat{\lambda^{*}} \mid x\right)}{q(\hat{\lambda} \mid x)}
$$

where $\hat{\lambda}^{*}$ and $\hat{\lambda}$ maximize $l^{*}(\lambda)$ and $l(\lambda)$ respectively, and $\Omega^{*}$ and $\Omega$ are the negatives of the inverses of the matrices of second derivatives of $l^{*}(\lambda)$ and $l(\lambda)$ (given in the Appendix C) at $\hat{\lambda}^{*}$ and $\hat{\lambda}$, respectively. Also, $q(\lambda \mid x)$ is the posterior p.d.f. of $\lambda$ given the data. Under the assumption that the parameters $k, a$ and $s$ are unknown, we consider the following priors on $k, a$ and $s$. The parameters $k$ and $s$ have the gamma priors with shape parameters $\theta_{1}, \theta_{2}$ and scale parameters $\eta_{1}^{-1}, \eta_{2}^{-1}$, respectively. Also, $a$ has a non-informative prior as $\frac{1}{a}$. The posterior p.d.f. of $k, a$ and $s$ given the data is given as

$$
\begin{aligned}
q(k, a, s \mid x) \propto & s^{n+\theta_{2}-1} k^{n+\theta_{1}-1} a^{n k-1} e^{-s\left(\eta_{2}-\sum_{i=1}^{n} x_{i}\right)} \\
& \times e^{-k\left(\eta_{1}+\sum_{i=1}^{n} \ln \left(e^{s x_{i}}+a-1\right)\right)-\sum_{i=1}^{n} \ln \left(e^{s x_{i}}+a-1\right)} .
\end{aligned}
$$

Here, we consider $\theta_{1}=\theta_{2}=3$ and $\eta_{1}=\eta_{2}=2$. We use this approximation to obtain Bayes estimators for the parameters $k$ and $a$ and $s$ using of $R$ software. Also, we used Newton-Raphson method by using of "nlm" function in $R$ software for optimization. Table 2 shows the Bayes estimates and the root of mean square error (RMSE) for various parameter values of the LED.

\subsection{Simulation}

Tables 3 and 4 provide the mean, median, variance, skewness, kurtosis and Shannon entropy of the LED for various values of $k, a$ and $s$. We used "actuar" and "moment"packages in $R$ software. These results are confirmed by (9) and (11). The results show that when $k$ or $s$ are increasing the mean, median and variance are decreasing and when $a$ is increasing, the mean, median and variance are increasing. 
Table 2. Bayes estimates and the root of MSE (RMSE) for various parameter values

\begin{tabular}{|c|c|c|c|c|c|c|c|c|}
\hline \multicolumn{3}{|c|}{ Actual values } & \multicolumn{2}{|c|}{ Estimates of $k$} & \multicolumn{2}{|c|}{ Estimates of $a$} & \multicolumn{2}{|c|}{ Estimates of $s$} \\
\hline$k$ & $a$ & $s$ & Mean & RMSE & Mean & RMSE & Mean & RMSE \\
\hline 0.25 & 0.25 & 0.25 & 0.2911 & 0.1801 & 0.2526 & 0.1210 & 0.3022 & 0.1344 \\
\hline 0.25 & 0.25 & 0.5 & 0.3228 & 0.1195 & 0.2635 & 0.2404 & 0.5750 & 0.1513 \\
\hline 0.25 & 0.5 & 0.25 & 0.3492 & 0.5676 & 0.5443 & 0.0979 & 0.2768 & 0.1818 \\
\hline 0.5 & 0.25 & 0.25 & 0.4897 & 0.0983 & 0.2620 & 0.2887 & 0.4213 & 0.1734 \\
\hline 1 & 0.5 & 0.25 & 0.7277 & 0.2168 & 0.5623 & 0.5456 & 0.6947 & 0.3383 \\
\hline 0.75 & 1 & 0.25 & 0.6045 & 0.4269 & 1.0582 & 0.4087 & 0.4302 & 0.2197 \\
\hline 0.25 & 0.75 & 1 & 0.6367 & 0.3400 & 0.8271 & 0.3901 & 0.7513 & 0.4184 \\
\hline 1.5 & 0.25 & 0.25 & 1.1031 & 0.1895 & 0.3624 & 0.9179 & 0.9083 & 0.5238 \\
\hline 0.25 & 1.5 & 0.5 & 0.5714 & 0.4340 & 1.4510 & 0.0911 & 0.4369 & 0.3840 \\
\hline 0.5 & 0.25 & 1.25 & 0.7141 & 0.0954 & 0.2528 & 1.0223 & 2.1486 & 0.3060 \\
\hline 0.5 & 0.25 & 2.25 & 0.9101 & 0.0903 & 0.2362 & 2.2252 & 2.2656 & 0.5234 \\
\hline 1 & 0.25 & 2 & 2.0003 & 0.0963 & 0.1976 & 0.9796 & 1.6518 & 1.7319 \\
\hline 0.5 & 2 & 0.25 & 0.6056 & 0.4978 & 1.8255 & 0.1790 & 0.3823 & 0.3081 \\
\hline
\end{tabular}

\section{Applications}

In this section, we fit the LED to two real data sets and show that the LED is more flexible in analyzing of the data than the previous analyzes and some other distributions. The first data from Raqab et al. (2008) are: $0.312,0.314,0.479,0.552,0.700,0.803,0.861,0.865,0.944,0.958,0.966,0.997$, 1.006, 1.021, 1.027, 1.055, 1.063, 1.098, 1.140, 1.179, 1.224, 1.240, 1.253, 1.270, $1.272,1.274,1.301,1.301,1.359,1.382,1.382,1.426,1.434,1.435,1.478,1.490$, $1.511,1.514,1.535,1.554,1.566,1.570,1.586,1.629,1.633,1.642,1.648,1.684$, 1.697, 1.726, 1.770, 1.773, 1.800, 1.809, 1.818, 1.821, 1.848, 1.880, 1.954, 2.012, $2.067,2.084,2.090,2.096,2.128,2.233,2.433,2.585,2.585$.

These data represent the tensile strength data measured in GPa for singlecarbon fibers. Alzaatreh and Knight (2013) fitted these data to the gammahalf normal, half normal, generalized half-normal and beta generalized half normal distributions. They indicated that the generalized half-normal and beta generalized half normal and gamma-half normal distributions each provide a good fit for data and among the three distributions, the gamma-half normal distribution provides the best fit for these data. We compare the LED with the generalized exponential distribution (GED), the gamma-half 
Table 3. Mean, Median, Variance, Skewness, Kurtosis and Shannon entropy for Some Values of $k, a$ and $s$

\begin{tabular}{|c|c|c|c|c|c|c|c|c|}
\hline$k$ & $\bar{a}$ & $\bar{s}$ & Mean & Median & Variance & Skewness & Kurtosis & Sh. E. \\
\hline \multirow[t]{4}{*}{0.5} & 0.5 & 0.5 & 3.1185 & 2.0087 & 15.6035 & 1.9698 & 7.7661 & 1.2687 \\
\hline & & 1 & 1.6481 & 0.90080 & 3.3389 & 2.5668 & 12.5013 & 1.3010 \\
\hline & & 2 & 0.8159 & 0.4308 & 0.7615 & 1.9341 & 11.1235 & 1.6667 \\
\hline & & 3 & 0.5393 & 0.3628 & 0.3572 & 2.1398 & 9.0394 & 2.1572 \\
\hline \multirow[t]{4}{*}{0.5} & 1 & 0.5 & 3.7796 & 2.8198 & 16.4038 & 1.8866 & 7.8411 & 1.4354 \\
\hline & & 1 & 2.2165 & 1.4155 & 4.5158 & 1.5401 & 4.4378 & 1.3010 \\
\hline & & 2 & 0.9907 & 0.7304 & 1.1542 & 1.5243 & 8.7126 & 1.3333 \\
\hline & & 3 & 0.6764 & 0.5497 & 0.3730 & 2.2319 & 9.1203 & 1.4906 \\
\hline \multirow[t]{4}{*}{0.5} & 2 & 0.5 & 4.6988 & 3.7738 & 15.5668 & 1.5488 & 6.1392 & 1.5187 \\
\hline & & 1 & 2.6032 & 1.9382 & 3.8661 & 1.6821 & 8.8523 & 1.3010 \\
\hline & & 2 & 1.2847 & 0.8954 & 1.0619 & 1.8601 & 7.1761 & 1.1667 \\
\hline & & 3 & 0.8674 & 0.6325 & 0.5522 & 1.7972 & 5.1673 & 1.1572 \\
\hline \multirow[t]{4}{*}{0.5} & 3 & 0.5 & 5.6513 & 5.1019 & 20.7863 & 1.1266 & 4.9095 & 1.5465 \\
\hline & & 1 & 2.7528 & 2.2410 & 5.2152 & 1.3944 & 4.6113 & 1.3010 \\
\hline & & 2 & 1.3641 & 1.0994 & 1.2648 & 2.0711 & 7.3325 & 1.1111 \\
\hline & & 3 & 0.9447 & 0.8047 & 0.6137 & 1.5036 & 6.8796 & 1.0461 \\
\hline \multirow[t]{4}{*}{1} & 0.5 & 0.5 & 1.3283 & 0.7551 & 3.0445 & 2.6249 & 9.4223 & 0.8010 \\
\hline & & 1 & 0.6879 & 0.3771 & 0.6233 & 2.5564 & 11.0976 & 1.0000 \\
\hline & & 2 & 0.3417 & 0.20042 & 0.1498 & 2.4982 & 11.8602 & 1.6990 \\
\hline & & 3 & 0.2549 & 0.1361 & 0.0640 & 2.2988 & 14.6936 & 2.5229 \\
\hline \multirow[t]{4}{*}{1} & 1 & 0.5 & 1.9837 & 1.2528 & 3.5541 & 1.8634 & 4.8570 & 1.0510 \\
\hline & & 1 & 1.0600 & 0.6596 & 0.9504 & 1.6785 & 7.9160 & 1.0000 \\
\hline & & 2 & 0.4878 & 0.3560 & 0.2433 & 2.0376 & 8.1892 & 1.1990 \\
\hline & & 3 & 0.3234 & 0.2277 & 0.1087 & 2.1171 & 6.1072 & 1.5229 \\
\hline \multirow[t]{4}{*}{1} & 2 & 0.5 & 2.8657 & 2.2705 & 6.2535 & 1.5594 & 9.7372 & 1.1760 \\
\hline & & 1 & 1.4169 & 1.0762 & 1.1733 & 1.0867 & 5.2483 & 1.0000 \\
\hline & & 2 & 0.6915 & 0.5883 & 0.3490 & 1.4217 & 7.8099 & 0.9490 \\
\hline & & 3 & 0.4434 & 0.3405 & 0.1681 & 1.3656 & 6.7946 & 1.0229 \\
\hline \multirow[t]{4}{*}{1} & 3 & 0.5 & 3.1279 & 3.0498 & 7.2099 & 0.9886 & 5.7094 & 1.2177 \\
\hline & & 1 & 1.5655 & 1.3980 & 1.6355 & 1.4784 & 5.0560 & 1.0000 \\
\hline & & 2 & 0.8385 & 0.7799 & 0.4351 & 1.1636 & 5.9379 & 0.8656 \\
\hline & & 3 & 0.5893 & 0.4519 & 0.1563 & 1.4726 & 4.0430 & 0.8562 \\
\hline
\end{tabular}


Table 4. Mean, Median, Variance, Skewness, Kurtosis and Shannon entropy for Some Values of $k, a$ and $s$

\begin{tabular}{|c|c|c|c|c|c|c|c|c|}
\hline $\bar{k}$ & $\bar{a} a$ & $\bar{s}$ & Mean & Median & Variance & Skewness & Kurtosis & Sh. E. \\
\hline \multirow[t]{4}{*}{2} & 0.5 & 0.5 & 0.6598 & 0.3812 & 0.5100 & 2.4139 & 21.4506 & 0.3333 \\
\hline & & 1 & 0.3330 & 0.1936 & 0.1428 & 2.2095 & 13.8428 & 0.6990 \\
\hline & & 2 & 0.1450 & 0.0944 & 0.0260 & 2.6158 & 12.2884 & 1.7313 \\
\hline & & 3 & 0.0969 & 0.0654 & 0.0135 & 2.9746 & 14.5280 & 2.8885 \\
\hline \multirow[t]{4}{*}{2} & 1 & 0.5 & 1.0836 & 0.7052 & 1.3511 & 2.2532 & 8.7881 & 0.6667 \\
\hline & & 1 & 0.5386 & 0.3444 & 0.2268 & 1.9310 & 7.0980 & 0.6990 \\
\hline & & 2 & 0.2586 & 0.1645 & 0.0686 & 1.5183 & 7.2673 & 1.0646 \\
\hline & & 3 & 0.1703 & 0.1051 & 0.0293 & 1.8606 & 5.1221 & 1.5552 \\
\hline \multirow[t]{4}{*}{2} & 2 & 0.5 & 1.4940 & 1.2056 & 2.0512 & 1.1961 & 7.4123 & 0.8333 \\
\hline & & 1 & 0.7475 & 0.6690 & 0.4453 & 1.4505 & 7.4357 & 0.6990 \\
\hline & & 2 & 0.3675 & 0.2890 & 0.1016 & 1.5627 & 4.5338 & 0.7313 \\
\hline & & 3 & 0.2727 & 0.2146 & 0.0579 & 1.9885 & 5.5808 & 0.8885 \\
\hline \multirow[t]{4}{*}{2} & 3 & 0.5 & 1.9448 & 1.7146 & 2.1895 & 0.9909 & 4.2033 & 0.8889 \\
\hline & & 1 & 0.9926 & 0.7790 & 0.5460 & 1.3771 & 4.5162 & 0.6990 \\
\hline & & 2 & 0.4843 & 0.3846 & 0.1491 & 0.9969 & 4.3084 & 0.6202 \\
\hline & & 3 & 0.3276 & 0.3027 & 0.0719 & 1.2928 & 5.0748 & 0.6663 \\
\hline \multirow[t]{4}{*}{3} & 0.5 & 0.5 & 0.4067 & 0.2391 & 0.1770 & 2.2094 & 19.7520 & 0.0739 \\
\hline & & 1 & 0.2155 & 0.1117 & 0.0537 & 2.8679 & 11.5258 & 0.5229 \\
\hline & & 2 & 0.0994 & 0.0674 & 0.0115 & 1.7575 & 9.4363 & 1.7218 \\
\hline & & 3 & 0.0600 & 0.0406 & 0.0065 & 5.4833 & 14.4411 & 3.0458 \\
\hline \multirow[t]{4}{*}{3} & 1 & 0.5 & 0.6498 & 0.4340 & 0.4968 & 0.9708 & 6.6093 & 0.4489 \\
\hline & & 1 & 0.3297 & 0.2167 & 0.1056 & 2.0249 & 8.0030 & 0.5229 \\
\hline & & 2 & 0.1645 & 0.1143 & 0.0250 & 1.9507 & 8.3112 & 0.9718 \\
\hline & & 3 & 0.1208 & 0.0775 & 0.0107 & 1.7890 & 10.1425 & 1.5458 \\
\hline \multirow[t]{4}{*}{3} & 2 & 0.5 & 1.0843 & 0.7629 & 0.8187 & 1.3843 & 3.7437 & 0.6364 \\
\hline & & 1 & 0.5725 & 0.4000 & 0.2183 & 1.3401 & 5.4144 & 0.5229 \\
\hline & & 2 & 0.2669 & 0.2145 & 0.0552 & 1.5032 & 5.4768 & 0.5968 \\
\hline & & 3 & 0.1771 & 0.1376 & 0.0244 & 1.5966 & 4.4785 & 0.7958 \\
\hline \multirow[t]{4}{*}{3} & 3 & 0.5 & 1.3642 & 1.2024 & 1.2186 & 1.3601 & 3.7515 & 0.6989 \\
\hline & & 1 & 0.7286 & 0.5959 & 0.3581 & 1.1269 & 6.3090 & 0.5229 \\
\hline & & 2 & 0.3613 & 0.2822 & 0.0723 & 0.8862 & 4.0228 & 0.4718 \\
\hline & & 3 & 0.2362 & 0.2059 & 0.0368 & 1.2138 & 5.5008 & 0.5458 \\
\hline
\end{tabular}


Table 5. Parameter estimates for the single carbon fibers

\begin{tabular}{lccccc}
\hline \hline Distribution & GED & GHD & LD & GD & LED \\
\hline Parameter estimation & $\hat{\alpha}=8.82$ & $\hat{\alpha}=2.8794$ & $\hat{k}=133.4542$ & $\hat{\alpha}=6.9969$ & $\hat{k}=1.3773$ \\
& $\hat{\beta}=0.55$ & $\hat{\beta}=3.1726$ & $\hat{a}=193.5781$ & $\hat{\beta}=0.2074$ & $\hat{a}=147.087$ \\
& & $\hat{\theta}=0.3934$ & & & $\hat{s}=3.1330$ \\
\hline AIC & 117.33 & 105.32 & 193.81 & 110.16 & $\mathbf{1 0 3 . 6 7}$ \\
AICc & 117.51 & 105.68 & 193.99 & 110.34 & $\mathbf{1 0 4 . 0 3}$ \\
BIC & 121.80 & 112.03 & 198.32 & 114.63 & $\mathbf{1 1 0 . 3 7}$ \\
HQIC & 119.10 & 110.87 & 195.63 & 111.93 & $\mathbf{1 0 6 . 4 5}$ \\
K-S & 0.9264 & 0.0865 & 0.6286 & 0.0883 & $\mathbf{0 . 0 3 9 5}$ \\
K-S $p$-value & $<0.0000$ & 0.9985 & $<0.0000$ & 0.6536 & $\mathbf{0 . 9 9 9 9}$ \\
\hline
\end{tabular}

normal distribution (GHD), the Lomax distribution (LD) and the gamma distribution (GD). The maximum likelihood estimates, Akaike Information Criterion (AIC), Consistent Akaike Information Criterion (AICc), Bayesian Information Criterion (BIC), Hannon-Quinn Information Criterion (HQIC), Kolmogorov-Smirnov test statistic (K-S) and $p$-value for the fitted distributions are reported in Table 5. The results in Table 5 show that the LD and GED are not good models for the data. The GHD and the GD are good models for the data. However, the LED has lowest values for the AIC, AICc, BIC and HQIC. Also, it has the most K-S $p$-value. Then the LED provides an excellent good fit to the data. The plot of the densities of LED, GHD and GD fitted to the data set in Figure 3 shows that the LED gives a better fit than the GHD and the GD. The linearity of the points in Q-Q plot suggests that the LED can be the appropriate model for the first data.

The second data set from Lawless (1982) has been analyzed by Kundu and Gupta (2008). The data are the number of million revolutions before failure for each of the 23 ball bearing in the life test and they are 17.88, $28.92,33,41.52,42.12,45.60,48.40,51.84,51.96,54.12,55.56,67.80,68.64$, $68.64,68.88,84.12,93.12,98.64,105.12,105.84,127.92,128.04,173.40$.

They analyzed the data using the generalized exponential distribution (GED). To check the validity of the model, they computed the Kolmogorov-Smirnov (K-S) distance between the empirical distribution function and the fitted distribution function when the parameters were obtained by different methods, 


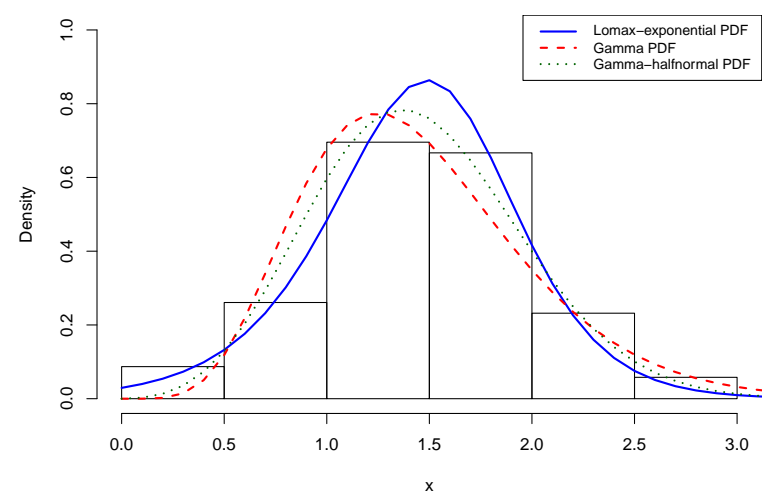

(a)

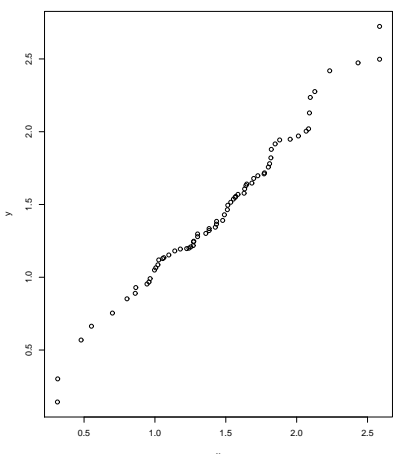

(b)

Figure 3. (a) Estimated densities of the LED, GD and GHD for the first data set. (b) The Q-Q plot of the LED for the first data set

namely by MLEs, exact Bayes estimators and approximate Bayes estimators (MCMC). They showed that the estimated general exponential distributions provides good fit to the given data. We fit the LED to these data and compare this distribution with the GED, exponential distribution (ED), LD and the GD. We computed Kolmogorov-Smirnov test statistic (K-S), K-S p-value, Cramer-Von Mises test statistics (C-VM) and C-VM $p$-value for the fitted distributions. We used "goftest" package in $R$ software. The results in Table (6) show that the LED, GED and GD are good models for the data but the LED fits these data better than the others. The plot of the densities of LED, GD and GRD fitted to the data set in Figure 4 shows that the LED gives a better fit than the others. The linearity of the points in Q-Q plot suggests that the LED can be the appropriate model for the second data. Also Figure 5 compares the estimated c.d.f's of the LED, GD and GED with the empricial c.d.f. of the second data set.

\section{Conclusions}

We introduced a new distribution, LED. It includes the exponential distribution and has a simple relation with the Lomax distribution. Some general properties were studied. The shapes of the LED can be approximately sym- 
Table 6. K-S distances and corresponding $p$-values for the number of milion revolution before failure for each of the 23 ball bearing in the life test

\begin{tabular}{cccccc}
\hline \hline Distribution & GED & ED & LD & GD & LED \\
\hline Parameter estimation & $\hat{\alpha}=5.2836$ & $\hat{\alpha}=0.0138$ & $\hat{k}=5780.61431$ & $\hat{\alpha}=4.0247$ & $\hat{k}=0.2231$ \\
& $\hat{\beta}=0.0323$ & & $\hat{a}=80.66048$ & $\hat{\beta}=17.9443$ & $\hat{a}=95.9975$ \\
& & & & & $\hat{s}=0.1209$ \\
\hline K-S & 0.1052 & 0.5129 & 1 & 0.1229 & $\mathbf{0 . 0 8 0 9}$ \\
K-S $p$-value & 0.9592 & $<0.0000$ & $<0.0000$ & 0.8778 & $\mathbf{0 . 9 9 8 2}$ \\
\hline C-VM & 0.0961 & 7.6651 & 7.2577 & 0.1622 & $\mathbf{0 . 0 2 5 6}$ \\
C-VM $p$-value & 0.6084 & $<0.0000$ & $<0.0000$ & 0.3562 & $\mathbf{0 . 9 8 9 7}$ \\
\hline
\end{tabular}

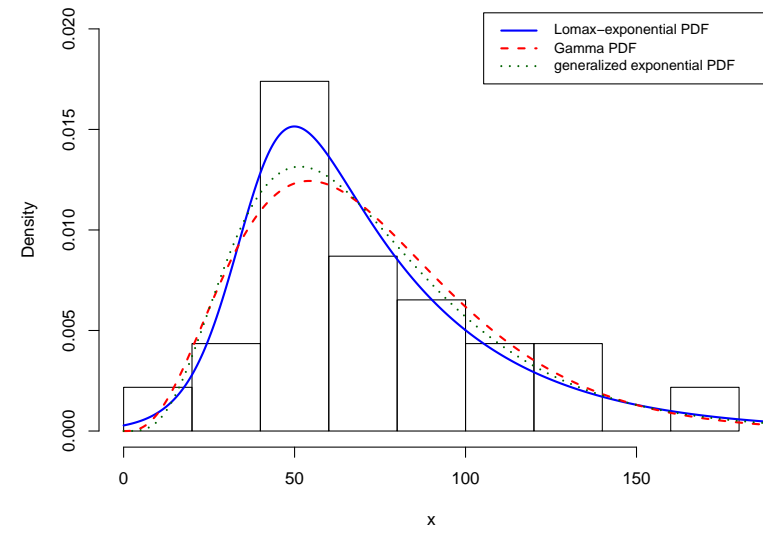

(a)

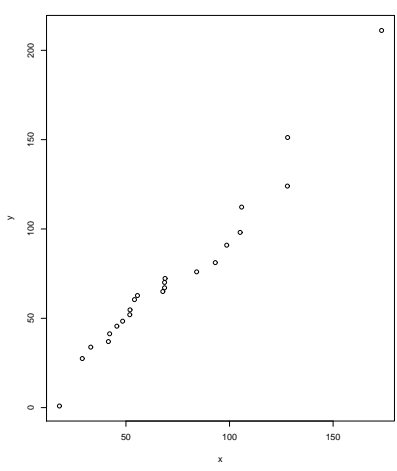

(b)

Figure 4. (a) Estimated densities of the LED, GD and GED for the second data set. (b) The Q-Q plot of the LED for the second data set

metric, skewed to the right and reversed J-shape. Then, it is a good model for skewed to right data. Simulations of this distribution were provided and they were compared with theoretical equations. The LED was fitted to two real data sets. It fitted the data better than previous analyzes.

\section{Acknowledgement}

The authors would like to thank the Editor and Referees for the constructive comments and illustrative suggestions, which greatly improved the quality 


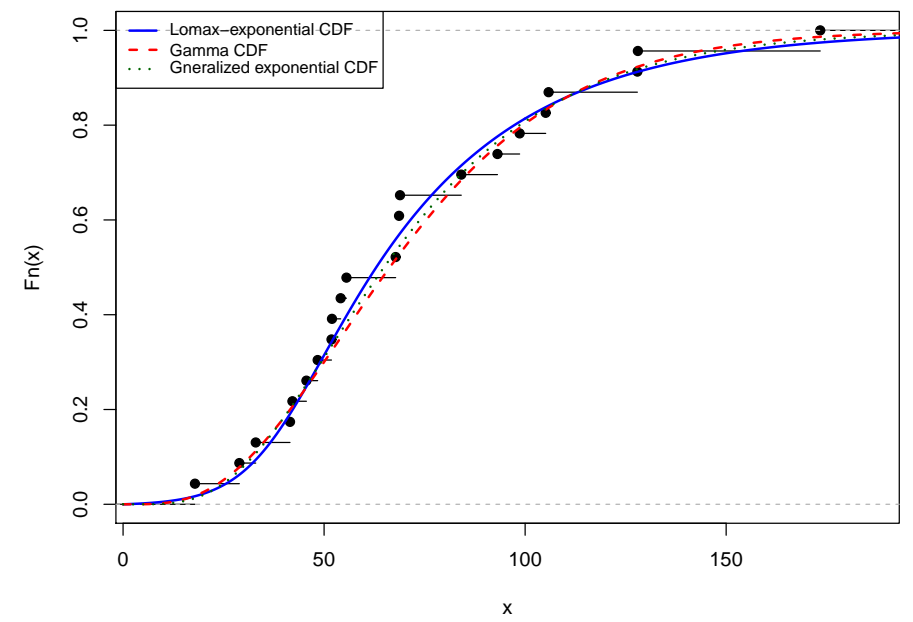

Figure 5. Estimated c.d.f. of the LED, GD and GED and empricial c.d.f. of the second data set

and presentation of the manuscript.

\section{References}

Alzaatreh, A., Famoye, F. and Lee, C. (2012). Gamma-Pareto Distribution and Its Applications, Journal of Modern Applied Statistical Methods, 11, 78-94.

Alzaatreh, A., Lee, C. and Famoye, F. (2013a). A New Method for Generating Families of Continuous Distributions, Metron, 71, 63-79.

Alzaatreh, A., Famoye, F. and Lee, C. (2013b). Weibull-Pareto Distribution and Its Applications, Communications in Statistics-Theory and Methods, 42, 1673-1691.

Alzaatreh, A., Famoye, F. and Lee, C. (2014). The Gamma-normal Distribution: Properties and Applications. Computational Statistics \& Data Analysis, 69, 67-80.

Alzaatreh, A. and Knight, K. (2013). On the Gamma-half Normal Distribution and Its Applications, Journal of Modern Applied Statistical Methods, 12, 103-119.

Arnold, B., Balakrishnan, N. and Nagaraja, H.N. (2008). First Course in Order Statistics, Philadephia, Siam publishers. 
Balakrishnan, N. and Basu, A.P. (1995). The Exponential Distribution, New York, Gordon and Breach.

Gupta, R.D. and Kundu, D. (1999a). Generalized Exponential Distribution, Australian \& New Zealand Journal of Statistics, 41, 173-188.

Gupta, R.D. and Kundu, D. (1999b). Generalized Exponential Distribution: Different Method of Estimations. Journal of Statistical Computation and Simulation, 00, 1-22.

Kundu, D. and Gupta, R.D. (2008). Generalized Exponential Distribution: Bayesian Estimations, Computational Statistics 83 Data Analysis, 52, 1873-1883.

Lawless, J.F. (1982). Statistical Models and Methods for Lifetime Data, New York, Wiley.

Lemonte, A. and Cordeiro, G. (2011). An Extended Lomax Distribution, Statistics, iFirst (4), 1-17.

Raqab, M., Madi, T. and Debasis, K. (2008). Estimation of $P(Y<X)$ for the 3- Parameter Generalized Exponential Distribution, Communications in Statistics-Theory and Methods, 37, 2854-2864.

Shannon, C.E. (1948). A Mathematical Theory of Communication. Bell System Technical Journal, 27, 379-432.

Tierney, L. and Kadane, J.B. (1986). Accurate Approximations for Posterior Moments and Marginal Densities, Journal of the American Statistical Association, 81, 82-86.

Torabi, H. and Montazeri, N.H. (2012). The Gamma-uniform Distribution and Its Applications, Kybernetika, 48, 16-30.

Torabi, H. and Montazeri, N.H. (2014). The Logistic-uniform Distribution and Its Application, Communications in Statistics-Theory and Methods, 7, 169-188.

\section{Appendix A}

The p.d.f. and c.d.f. of distributions applied in the paper are given in the following.

- The p.d.f. and c.d.f. of the Pareto type 1 distribution are respectively defined for $t>a, k, a>0$ as

$$
f(t)=\frac{k a^{k}}{t^{k+1}}
$$


and

$$
F(t)=1-\left(\frac{a}{t}\right)^{k}
$$

- The p.d.f. and c.d.f. of the generalized exponential distribution, respectively, are given for $t>0, \alpha, \lambda>0$

$$
f(t)=\alpha \lambda(1-\exp (-\lambda t))^{\alpha-1} \exp (-\lambda t)
$$

and

$$
F(t)=(1-\exp (-\lambda t))^{\alpha} .
$$

- The p.d.f. and c.d.f. of the gamma distribution, respectively, for $t>$ $0, \alpha, \beta>0$ are

$$
f(t)=\frac{1}{\Gamma(\alpha) \beta^{\alpha}} t^{\alpha-1} e^{-\frac{t}{\beta}}
$$

and

$$
F(t)=\frac{\gamma\left(\alpha, \frac{t}{\beta}\right)}{\Gamma(\alpha)} .
$$

- The gamma-halfnormal distribution is defined with p.d.f. and c.d.f. as follow

$$
f(t)=\frac{\sqrt{2}}{\theta \sqrt{\pi}} e^{-\frac{t^{2}}{2 \theta^{2}}}\left[-\log \left\{2 \Phi\left(-\frac{t}{\theta}\right)\right\}\right]^{\alpha-1}\left\{2 \Phi\left(-\frac{t}{\theta}\right)\right\}^{\frac{1}{\beta}-1}
$$

and

$$
F(t)=\frac{\gamma\left(\alpha,-\beta^{-1} \log \left(2 \phi\left(-\frac{t}{\theta}\right)\right)\right)}{\Gamma(\alpha)},
$$

where $t>0, \alpha, \beta, \theta>0$. 


\section{Appendices B and C}

The second derivatives of $\log L(k, a, s)$ are given as

$$
\begin{gathered}
\frac{\partial^{2} L}{\partial a^{2}}=\frac{-n k}{a^{2}}+\sum_{i=1}^{n} \frac{(k+1)}{\left(e^{s x_{i}}+a-1\right)^{2}}, \\
\frac{\partial^{2} L}{\partial k^{2}}=-\frac{n}{k^{2}}, \\
\frac{\partial^{2} L}{\partial s^{2}}=\frac{-n}{s^{2}}-\sum_{i=1}^{n} \frac{(a-1)(k+1) x_{i}^{2} e^{s x_{i}}}{\left(e^{s x_{i}}+a-1\right)^{2}}, \\
\frac{\partial^{2} L}{\partial a \partial k}=\frac{n}{a}-\sum_{i=1}^{n} \frac{1}{e^{s x_{i}}+a-1} \\
\frac{\partial^{2} L}{\partial a \partial s}=\sum_{i=1}^{n} \frac{(k+1) x_{i} e^{s x_{i}}}{\left(e^{s x_{i}}+a-1\right)^{2}},
\end{gathered}
$$

and

$$
\frac{\partial^{2} L}{\partial k \partial s}=-\sum_{i=1}^{n} \frac{x_{i} e^{s x_{i}}}{e^{s x_{i}}+a-1}
$$

The second derivatives of $l(k, a, s)$ are given as

$$
\begin{aligned}
& \frac{\partial^{2} l}{\partial a^{2}}=\frac{1}{n}\left\{\frac{-(n k-1)}{a^{2}}+(k+1) \sum_{i=1}^{n}\left(e^{s x_{i}}+a-1\right)^{-1}\right\}, \\
& \frac{\partial^{2} l}{\partial s^{2}}=\frac{1}{n}\left\{-\frac{n+\theta_{2}-1}{s^{2}}-(k+1) \sum_{i=1}^{n} \frac{x_{i}^{2} e^{s x_{i}}(a-1)}{\left(e^{s x_{i}}+a-1\right)^{2}}\right\},
\end{aligned}
$$




$$
\begin{gathered}
\frac{\partial^{2} l}{\partial k^{2}}=-\frac{n+\theta_{1}-1}{n k^{2}}, \\
\frac{\partial^{2} l}{\partial a \partial s}=\frac{(k+1)}{n} \sum_{i=1}^{n} \frac{x_{i} e^{s x_{i}}}{\left(e^{s x_{i}}+a-1\right)^{2}}, \\
\frac{\partial^{2} l}{\partial a \partial k}=\frac{1}{n}\left\{\frac{n}{a}-\sum_{i=1}^{n}\left(\frac{1}{e^{s x_{i}}+a-1}\right)\right\},
\end{gathered}
$$

and

$$
\frac{\partial^{2} l}{\partial s \partial k}=-\frac{1}{n} \sum_{i=1}^{n} \frac{x_{i} e^{s x_{i}}}{e^{s x_{i}}+a-1} .
$$

\section{Nasrin Hami Golzar}

Department of Statistics,

Faculty of Mathematical Scince,

University of Mohaghegh Ardabili,

Ardabil, Iran.

email: nasrin.hami@uma.ac.ir

\section{Masoud Ganji}

Department of Statistics,

Faculty of Mathematical Scince,

University of Mohaghegh Ardabili,

Ardabil, Iran.

email:mganji@uma.ac.ir

\section{Hossein Bevrani}

Department of Statistics,

Faculty of Mathematical Scince,

University of Tabriz,

Tabriz, Iran.

email: bevrani@tabrizu.ac.ir 